\title{
The role of the transcription factor ETV5 in insulin exocytosis
}

\author{
Ruth Gutierrez-Aguilar • Dong-Hoon Kim - Marina Casimir • Xiao-Qing Dai • \\ Paul T. Pfluger • Jongsun Park • April Haller • Elizabeth Donelan • Jisoo Park • \\ David D'Alessio • Stephen C. Woods • Patrick E. MacDonald • Randy J. Seeley
}

Received: 15 July 2013 / Accepted: 9 October 2013 / Published online: 5 November 2013

(C) Springer-Verlag Berlin Heidelberg 2013

\begin{abstract}
Aims/hypothesis Genome-wide association studies have revealed an association of the transcription factor ETS variant gene 5 (ETV5) with human obesity. However, its role in glucose homeostasis and energy balance is unknown.

Methods Etv5 knockout (KO) mice were monitored weekly for body weight (BW) and food intake. Body composition was measured at 8 and 16 weeks of age. Glucose metabolism
\end{abstract}

Electronic supplementary material The online version of this article (doi:10.1007/s00125-013-3096-5) contains peer-reviewed but unedited supplementary material, which is available to authorised users.

R. Gutierrez-Aguilar · A. Haller · E. Donelan • D. D’Alessio ·

R. J. Seeley $(\square)$

Department of Internal Medicine, University of Cincinnati,

2170 East Galbraith Road, Cincinnati, OH 45237, USA

e-mail: randy.seeley@uc.edu

\section{D.-H. Kim}

Department of Pharmacology, Korea University College of Medicine, Seoul, South Korea

M. Casimir $\cdot$ X.-Q. Dai $・$ P. E. MacDonald

Department of Pharmacology, University of Alberta,

Edmonton, AB, Canada

M. Casimir $\cdot$ X.-Q. Dai $・$ P. E. MacDonald

Alberta Diabetes Institute, University of Alberta,

Edmonton, AB, Canada

P. T. Pfluger

Institute for Diabetes and Obesity, Helmholtz Centre Munich,

Neuherberg, Germany

J. Park $\cdot$ J. Park

Department of Pharmacology, College of Medicine,

Chungnam National University, Daejeon, South Korea

S. C. Woods

Department of Psychiatry and Behavioral Neuroscience,

University of Cincinnati, Cincinnati, OH, USA was studied, and glucose-stimulated insulin secretion was measured in vivo and in vitro.

Results Etv5 KO mice are smaller and leaner, and have a reduced BW and lower fat mass than their wild-type controls on a chow diet. When exposed to a high-fat diet, $\mathrm{KO}$ mice are resistant to diet-induced BW gain. Despite a greater insulin sensitivity, $\mathrm{KO}$ mice have profoundly impaired glucose tolerance associated with impaired insulin secretion. Morphometric analysis revealed smaller islets and a reduced beta cell size in the pancreatic islets of Etv5 KO mice. Knockdown of ETV5 in an insulin-secreting cell line or beta cells from human donors revealed intact mitochondrial and $\mathrm{Ca}^{2+}$ channel activity, but reduced insulin exocytosis.

Conclusion/interpretation This work reveals a critical role for ETV5 in specifically regulating insulin secretion both in vitro and in vivo.

Keywords Beta cell · ETV5 - Genome-wide association study · Glucose homeostasis · Insulin exocytosis · Insulin secretion $\cdot$ Insulin sensitivity $\cdot$ Transcription factor

$\begin{array}{ll}\text { Abbreviations } \\ \text { BG } & \text { Blood glucose } \\ \text { BW } & \text { Body weight } \\ \text { DIO } & \text { Diet-induced obesity } \\ \text { EE } & \text { Energy expenditure } \\ \text { ETS } & \text { E-twenty-six } \\ \text { ETV5 } & \text { ETS variant gene } 5 \\ \text { FGF } & \text { Fibroblast growth factor } \\ \text { GSIS } & \text { Glucose-stimulated insulin secretion } \\ \text { GTT } & \text { Glucose tolerance test } \\ \text { GWAS } & \text { Genome-wide association studies } \\ \text { HFD } & \text { High-fat diet } \\ \text { ITT } & \text { Insulin tolerance test } \\ \text { KO } & \text { Knockout } \\ \text { MAPK } & \text { Mitogen-activated protein kinase }\end{array}$




$\begin{array}{ll}\text { MUNC-18 } & \text { Mammalian uncoordinated-18 } \\ \text { NS } & \text { Non-silencing } \\ \text { PEA3 } & \text { Polyomavirus enhancer activator 3 } \\ \text { siRNA } & \text { Small interfering RNA } \\ \text { SNAP25 } & \text { Synaptosomal-associated protein 25 } \\ \text { SNARE } & \begin{array}{l}\text { Soluble N-ethylmaleimide-sensitive factor } \\ \text { attachment protein receptor }\end{array} \\ \text { TMRE } & \text { Tetramethylrhodamine ethyl ester } \\ \text { VAMP2 } & \text { Vesicle-associated membrane protein 2 } \\ \text { WT } & \text { Wild-type }\end{array}$

\section{Introduction}

ETV5 (E-twenty-six variant gene 5, also known as ETSrelated protein, ERM) is a transcription factor that belongs to the polyomavirus enhancer activator 3 (PEA3) group of the 28 member E-twenty-six (ETS) family [1]. ETS factors are ubiquitously expressed in developing and adult mammalian tissues [2]. They can act as either transcriptional activators or repressors of multiple genes that regulate biological processes such as cell proliferation, differentiation, apoptosis and cellcell or cell-matrix interactions [3, 4].

ETS factors are active in diverse endocrine systems including the pituitary, thyroid and mammary glands, and the prostate, ovary, testis and pancreas [5]. PEA3 group transcription factors are nuclear effectors of growth factor signalling cascades. Fibroblast growth factor 10 (FGF10) regulates ETV5 in the lung and pancreas [6,7]. Overexpression of FGF10 in the pancreas leads to progenitor arrest, organ hyperplasia and inhibition of terminal differentiation [8], as well as elevated ETV5 levels [6]. ETV5 is highly expressed throughout pancreatic development, specifically in the distal epithelial cells [6].

Genome-wide association studies (GWASs) have revealed an association of ETV5 with human obesity in multiple populations $[9,10]$. However, a possible role of ETV5 in regulating metabolic variables has not been reported, highlighting the difficulty of determining the specific functions associated with genes identified in GWAS approaches.

Etv5-null mice were consequently used to assess metabolic parameters and the implications of ETV5 for metabolism. Etv 5 knockout (KO) mice are lean and resistant to dietinduced obesity (DIO) and, despite being lean, are severely glucose intolerant and hypoinsulinaemic. The data suggest an important cell-autonomous role for ETV5 in insulin exocytosis from beta cells.

\section{Methods}

Animals The generation of Etv $5 \mathrm{KO}$ mice has been described elsewhere [11]. KO and wild-type (WT) male littermates were derived from breeding heterozygous Etv 5 mice, and all comparisons are with littermates. Mice were housed individually and maintained on a $12 \mathrm{~h} / 12 \mathrm{~h}$ light/dark cycle. Eight-week-old animals were fed ad libitum with either a high-fat butter oil-based diet (HFD 45\%; Research Diets, New Brunswick, NJ, USA) or standard chow (HarlanTeklad, Indianapolis, IN, USA) for 8 weeks. Body weight (BW) and food intake were measured weekly. All procedures were approved by the University of Cincinnati Institutional Animal Care and Use Committee.

Body length and body composition Body length, measured from nose to anus, was determined in animals at 8 weeks of age. Body composition was assessed by nuclear magnetic resonance using an EchoMRI analyser (EchoMedical Systems, Houston, TX, USA) at 8 and 16 weeks of age.

Glucose tolerance and insulin tolerance tests Oral glucose tolerance tests (GTTs; $1.5 \mathrm{mg} / \mathrm{g} \mathrm{BW}$ ) were performed in 12-week-old mice after a $4 \mathrm{~h}$ fast. Blood glucose (BG) was measured at 0, 15, 30, 45, 60 and 120 min using Accu-Chek glucometers (Roche, Indianapolis, IN, USA). For the insulin tolerance tests (ITTs), mice that had been fasted for $4 \mathrm{~h}$ were administered human insulin (1 U/kg i.p.), and the glucose level was assessed at $0,15,30,45$ and $60 \mathrm{~min}$.

Insulin and C-peptide analysis Insulin levels were determined using the mouse endocrine LINCOplex kit (MENDO-75K; Linco Research, MI, USA). C-peptide was measured using the ALPCO mouse ELISA kit (ALPCO Diagnostics, Salem, NH, USA). Total pancreatic insulin extraction was performed by homogenising the whole pancreas in acid ethanol $(1.5 \% \mathrm{HCl}$ in $70 \%$ ethanol).

Histology, morphometric analysis and pancreatic immunohistochemistry Pancreases were fixed in $4 \%$ formalin and embedded in paraffin, sliced $(5 \mu \mathrm{m})$ with a separation of at least $150 \mu \mathrm{m}$, and mounted on slides. The distribution of islet size was determined by the relative frequencies of specific islet sizes from ten slides stained with haematoxylin and eosin (100 islets per animal). Islet areas were summated for the entire section, and the fractional area was calculated by dividing by total pancreatic area for that section. Beta cell mass was calculated from the relative cross-sectional beta cell area and total pancreatic mass.

For islet composition studies, immunohistochemistry was performed on three slides with a separation of $450 \mu \mathrm{m}$. Antigen retrieval was performed using a citrate buffer. The primary antibodies used were guinea pig anti-insulin (Abcam, Cambridge, MA, USA) at a dilution of 1:250 and rabbit antiglucagon antibody (Millipore, Billerica, MA, USA) at a dilution of 1:5,000 in 5\% bovine serum. The secondary antibodies were FITC anti-guinea pig IgG (Jackson ImmunoResearch Labs, 
West Grove, PA, USA) and Alexa Fluor 568 anti-rabbit IgG (Invitrogen, Life Technologies, Grand Island, NY, USA) at a dilution 1:1,000. Sections were covered with two drops of DAPI-Vectashield solution (Vector Laboratories, Burlingame, CA, USA). The positive staining areas were measured using AxioVision software (http://microscopy.zeiss.com/microscopy/ en_de/products/microscope-software/axiovision-for-biology. html\#Downloads). The number of nuclei of alpha and beta cells in each islet were counted and expressed as the percentage in the islet, or as the number of nuclei in a fixed area.

Glucose-stimulated insulin secretion from INS-1-cells Glucosestimulated insulin secretion (GSIS) from cultured INS-1 cells $(832 / 13)$ was carried out as previously described [12]. Cells were transfected with either Etv5 siRNA (Thermo Scientific, Rockford, IL, USA) or non-silencing (NS) siRNA (Qiagen, Valencia, CA, USA) for $72 \mathrm{~h}$. Cells were then washed, followed by a $2 \mathrm{~h}$ preincubation in $2 \mathrm{ml}$ of the Hanks' Buffered Salt Solution (HBSS) buffer. Insulin secretion was then measured in $1 \mathrm{~h}$ static incubations of cells in $2 \mathrm{ml}$ of HBSS containing the glucose concentrations indicated in the figure legends. Supernatant fractions from each well were collected for insulin assay. The total cellular insulin content was calculated as described elsewhere [12]. Insulin was measured with an ELISA kit (CrystalChem, Downers Grove, IL, USA).

INS-1 cell mitochondrial membrane potential and single-cell electrophysiology Mitochondrial membrane potential was measured using the fluorescent probe tetramethylrhodamine ethyl ester (TMRE; Invitrogen, Life Technologies, Grand Island, NY, USA), with the excitation and emission filters set at $549 \mathrm{~nm}$ and $574 \mathrm{~nm}$, respectively. Transduced cells or dispersed islets were loaded with $100 \mathrm{nmol} / \mathrm{l} \mathrm{TMRE}$ for $20 \mathrm{~min}$ at $37^{\circ} \mathrm{C}$ in Krebs-Ringer HEPES buffer (KRBH). After two serial washes, the effect of $15 \mathrm{mmol} / 1$ glucose (added on top of the basal $2.5 \mathrm{mmol} / \mathrm{l}$ ) was assessed, and fluorescence recordings were obtained every $20 \mathrm{~s}$. The results were normalised as the percentage stimulated vs $2.5 \mathrm{mmol} / \mathrm{l}$ glucose (using ImageJ software, http://rsb.info.nih.gov/ij/download.html).

To measure single-cell exocytosis and $\mathrm{Ca}^{2+}$ currents, the standard whole-cell technique with the sine+DC lock-in function of an EPC10 amplifier and Patchmaster software (HEKA-Electronics, http://www.heka.com/) was performed at $32-35^{\circ} \mathrm{C}$, as described elsewhere [13]. The concentration of glucose in the bath solution was either 2.5 or $15 \mathrm{mmol} / \mathrm{l}$, as indicated. The exocytosis of human beta cells was performed as previously described [13], and human beta cells were positively identified by insulin immunostaining. This procedure was approved by the Human Research Ethics Board of the University of Alberta. Cells were transfected with either ETV5 siRNA (Thermo Scientific) or NS siRNA (Qiagen) for $72 \mathrm{~h}$.
Gene expression Total RNA was extracted using a Qiagen miniprep RNA extraction kit. An iScript cDNA synthesis kit (Bio-Rad Laboratories, Foster City, CA, USA) was used. Quantitative RT-PCR was performed using a TaqMan 7900 sequence detection system and TaqMan gene expression assays (Applied Biosystems, Life Technologies). Relative mRNA expression for Etv5 (Rn00465814_g1), Snap25 (Rn00578534_m1), Munc-18 (Rn00564767_m1) (also known as Stxbp2), syntaxin 1A (Rn00587278_m1), Vamp2 (Rn01465442_m1), synaptotagmin VII (Rn00572234_m1) and synaptotagmin IX (Rn00584114_m1) was calculated relative to the housekeeping gene $L 32$ (Rn00820748_g1), using the $\Delta \Delta \mathrm{C}_{\mathrm{t}}$ method. The values are expressed as a percentage of the control siRNA in transfected INS-1 cells stimulated with $15 \mathrm{mmol} / \mathrm{l}$ glucose for $1 \mathrm{~h}$.

Western blots Protein extracts were obtained using RIPA buffer (Thermo Scientific) and run in SDS-PAGE gels. The antibodies used were: ETV5 (sc-22807, dilution 1:500) and Snap 25 (sc-7538, dilution 1:1,000) (Santa Cruz Biotechnology, Dallas, TX, USA); synaptotagmin IX (Cat. No. 105053, Synaptic Systems, Göttingen, Germany; dilution 1:5,000); and $\beta$-actin (4967, Cell Signaling, Billerica, MA, USA; dilution 1:5,000).

Metabolic phenotyping The energy expenditure (EE) and home-cage activity of WT and Etv5 KO mice were studied using an automated combined indirect calorimetry system (TSE Systems, Bad Homburg, Germany), as previously described [14]. After the mice had been acclimatised to the calorimetry cages, the RQ and EE were determined every 60 min for three light/dark phases. Home-cage locomotor activity was determined using a multidimensional infrared light beam system, with activity expressed as total beam breaks per $24 \mathrm{~h}$.

Histology and morphometric analysis of adipose tissue Haematoxylin and eosin-stained sections of epididymal fat were used.

Statistical analysis Data are presented as means \pm SEM. Statistical calculations were carried out using GraphPad Prism 5 (GraphPad Software, San Diego, CA, USA). The statistical significance of differences was determined by two-way ANOVA, followed by Bonferroni's multiple comparisons test or $t$ test analysis, as appropriate. A value of $p<0.05$ was considered statistically significant.

\section{Results}

Reduced BW, fat mass and food intake in Etv5 KO mice The transcription factor ETV5 has been implicated in the development of several tissues and, relative to their WT littermates, $\mathrm{KO}$ mice have previously been reported to have 
reduced BW throughout their lifespan $[11,15]$. In this study, we observed a significantly reduced body length at 8 weeks of age $(8.97 \pm 0.09$ vs $9.34 \pm 0.10 \mathrm{~cm}$; see electronic supplementary material [ESM] Fig. 1a, b).

When maintained on a chow diet, the KO mice had an $18 \%$ reduction in BW at 8 weeks of age $(18.06 \pm 1.06$ vs $21.97 \pm$ $1.38 \mathrm{~g})$ and a $26 \%$ reduction at 16 weeks of age $(19.40 \pm 0.84$ vs $26.30 \pm 1.06 \mathrm{~g}$ ) (Fig. 1a). Consistent with this, both fat and lean mass were significantly reduced in $\mathrm{KO}$ mice on a chow diet at both 8 and 16 weeks of age (Fig. 1b). When maintained on an HFD, the Etv $5 \mathrm{KO}$ mice were resistant to DIO and had a significant reduction in the amount epididymal white adipose
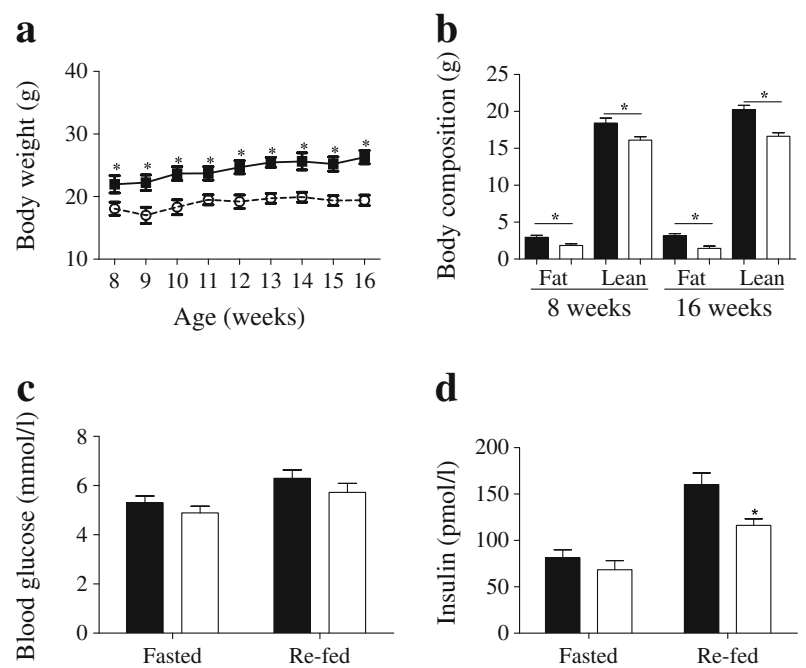

d
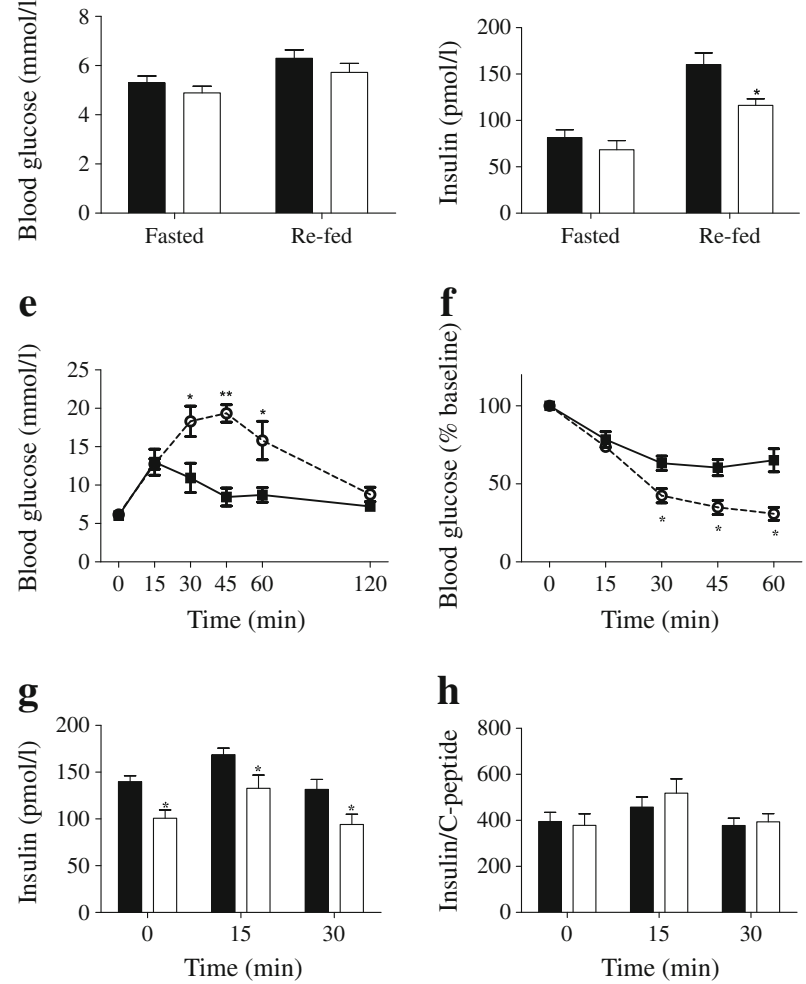

Fig. 1 Characterisation of BW, body composition and glucose homeostasis. (a) BW. (b) Body composition at 8 and 16 weeks of age. (c) Glucose levels after a $12 \mathrm{~h}$ fast and after $2 \mathrm{~h}$ of refeeding. (d) Insulin levels after $12 \mathrm{~h}$ of fasting and after $2 \mathrm{~h}$ of refeeding. (e) GTT. (f) ITT. (g) Insulin levels during the GTT. (h) Ratio of insulin to C-peptide levels (pmol $/ \mathrm{pmol}$ ) during the GTT. Animals were fed a chow diet. WT, $n=10$; $\mathrm{KO}, n=8$. All values are given as means $\pm \mathrm{SEM}$ from two-way ANOVA. Black symbols and columns, WT; white symbols and columns, Etv5 KO mice. ${ }^{*} p<0.05, * * p<0.01$ tissue (even when normalised by BW; data not shown) and smaller adipocytes than their WT littermates (ESM Fig. 1c-e, g). Food intake and total locomotor activity were reduced in KO mice compared with their WT littermates (ESM Fig. 1f, h). Indirect calorimetry revealed similar EE and RQ in WT and KO mice (ESM Fig. 1i, j; EE normalised to lean mass). Core body temperature was measured at several time points during the light/dark cycle and did not differ between genotypes (ESM Fig. 1k).

Impaired glucose homeostasis in Etv 5 KO mice Because BW affects glucose homeostasis, we evaluated the role of ETV5 in this respect. After a $12 \mathrm{~h}$ fast, BG and plasma insulin values were similar for both genotypes (Fig. 1c, d). However, after $2 \mathrm{~h}$ of refeeding, Etv $5 \mathrm{KO}$ mice had significantly lower plasma insulin (Fig. 1d).

At 12 weeks of age, the mice were given a GTT. Basal BG levels did not differ between WT and KO mice fed chow. Glucose levels were significantly increased in Etv $5 \mathrm{KO}$ compared with WT mice during the GTT and remained relatively elevated for $60 \mathrm{~min}$. By $120 \mathrm{~min}$, BG levels had returned to baseline in both genotypes (Fig. 1e). The same results were observed when an intraperitoneal GTT was performed (data not shown). Thus, despite their reduced BW, Etv 5 KO mice were glucose intolerant. Insulin levels during the GTT were $29 \%$ lower in the KO mice at baseline and were significantly lower than levels in WT mice after the glucose load (Fig. 1g). Ratios of insulin to C-peptide were similar for both genotypes (Fig. 1h), indicating that the difference in insulin levels is likely to be due to reduced secretion rather than an altered processing of proinsulin or an increased insulin breakdown. To determine whether glucose intolerance was caused by insulin resistance in the peripheral tissues, we performed an ITT. Etv5 $\mathrm{KO}$ mice had faster glucose clearance (Fig. 1f), consistent with increased insulin sensitivity.

In sum, Etv5 KO mice have increased insulin sensitivity and normal basal BG levels; however, they also have pronounced glucose intolerance accompanied by impaired insulin secretion.

Decreased beta cell size in Etv5 KO mice Pancreatic weight was significantly reduced in Etv $5 \mathrm{KO}$ mice (Fig. 2a). However, when normalised by BW, the difference disappeared (data not shown). To assess whether the hypoinsulinaemia in Etv5 KO mice was due to a reduced beta cell mass, we performed a histological analysis of the whole pancreas. Haematoxylin and eosin-stained pancreatic sections revealed a reduced number of islets per area of pancreas (Fig. 2b). The beta cell mass showed a trend towards reduction (Fig. 2c). There was a greater proportion of smaller islets in Etv 5 KO compared with WT mice (Fig. 2d), with the average islet size reduced by $32 \%$ (Fig. 2e), even when normalised to the total pancreatic area. 

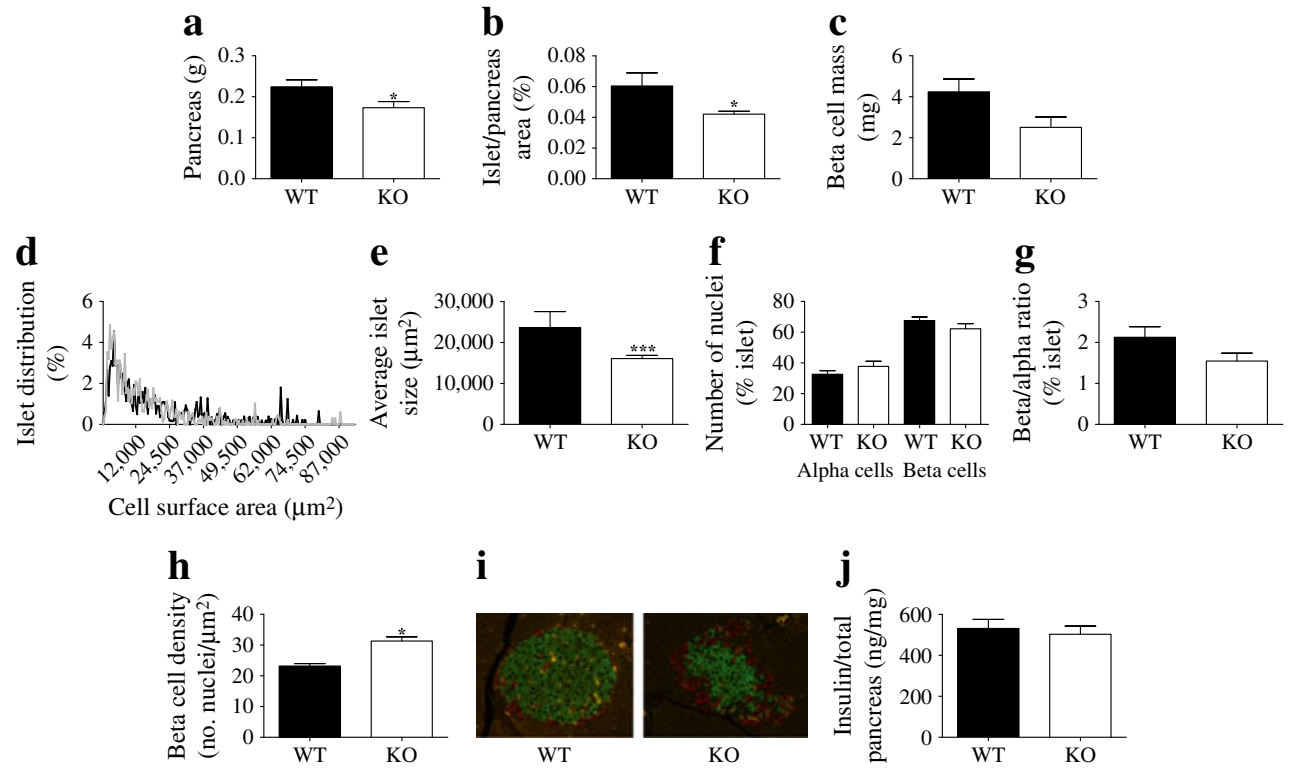

Fig. 2 Histological analysis of the pancreases. (a) Pancreas weight. (b) Islet size normalised by pancreas area. (c) Beta cell mass. (d) Islet distribution size; black, WT; grey, KO. (e) Average islet size. (f) Alpha and beta cell number. (g) Beta/alpha cell ratio. (h) Beta cell density was assessed by counting the number of nuclei in an islet area of $3,500 \mu^{2}$. (i)

Islet immunohistochemistry: anti-insulin (green) and anti-glucagon (red). WT, $n=4$; KO, $n=4$. (j) Total insulin extracted from whole pancreases normalised by total protein. WT, $n=6 ; \mathrm{KO}, n=4$. All values are given as means \pm SEM from $t$ test analysis. Black symbols and columns, WT; white symbols and columns, Etv5 KO mice. ${ }^{*} p<0.05,{ }^{* * *} p<0.001$

In addition, insulin and glucagon immunostaining revealed a trend towards a reduced beta to alpha cell ratio in Etv $5 \mathrm{KO}$ mice compared with their WT littermates (Fig. 2f, g). Insulin-positive cells were more densely packed in the Etv 5 KO islets (Fig. 2h). Representative islets of WT and Etv5 KO mice are depicted in Fig. 2i.

To determine whether decreased beta cell size affected the total amount of insulin produced by the pancreas, we extracted total insulin from the whole pancreas. No differences were found between genotypes (Fig. 2j), implying normal insulin production.

Thus, although there is a defect in islet morphology, the amount of total insulin is similar between genotypes. However, when the animals are challenged with glucose, insulin secretion is reduced in the Etv $5 \mathrm{KO}$ mice, suggesting a possible role of ETV5 in insulin secretion.

Impairment of GSIS in ETV5 knockdown INS-1 cells ETV5 has been linked to the development of different organ systems, including the pancreas $[6,7]$. To assess the loss of function of ETV5 while minimising any developmental impact, we moved to cell systems where we could use siRNA to knock down ETV5 production and analysed GSIS in a cellautonomous manner. We transfected insulin-secreting INS-1 cells with Etv 5 siRNA, achieving a $60 \%$ knockdown. No reduction of Etv 5 mRNA was observed in the presence of NS siRNA. The total insulin content was similar in cells treated with NS or Etv5 siRNA (Fig. 3a). However, insulin release stimulated by $15 \mathrm{mmol} / \mathrm{l}$ glucose was markedly reduced following knockdown of ETV5 (Fig. 3b). This was

not due to impaired mitochondrial function as the mitochondrial membrane potential responses to glucose, analysed by TMRE fluorescence, revealed no difference between the NS control and Etv5 siRNA groups (Fig. 3c).

We next assessed insulin secretion by the exocytotic response of single INS-1 cells. Use of these cell lines allows for knocking down of ETV5 after the cell has fully developed, and therefore minimises the developmental impact of the loss of function that occurs in the mouse model. Insulin exocytosis was assessed via increases in cell capacitance during a series of ten membrane depolarisations from -70 to $0 \mathrm{mV}$. Exocytotic responses at low $(2.5 \mathrm{mmol} / \mathrm{l})$ glucose were low, and did not differ between the NS control $(n=24)$ and the Etv 5 siRNA $(n=22)$ groups (Fig. 3d, e). Following glucose stimulation (15 mmol/1 for 10-15 $\mathrm{min}$ ), however, the total exocytotic response of the NS control cells was increased 4.3-fold $(n=28, p<0.001)$. In contrast, the exocytotic response of the Etv5 siRNA cells $(n=29)$ was significantly lower than in the controls $(p<0.001)$ (Fig. 3d, e). This difference is not likely to be due to altered $\mathrm{Ca}^{2+}$ channel activity, since voltage-dependent $\mathrm{Ca}^{2+}$ currents did not differ between groups $(n=33, \mathrm{NS}$, and 28 , Etv5 siRNA) (Fig. 3f, g). These data identify a defect in $\mathrm{Ca}^{2+}$-stimulated insulin exocytosis in the absence of ETV5.

There are always concerns about whether cell lines reflect the biology of specific cell types. Therefore, to determine whether these results generalise to human beta cells, we examined the effect of knocking down ETV5 in human beta cells from two different donors. Again, this approach allows an assessment of the cell-autonomous role of ETV5 with less concern for the developmental effects of the loss of function. 
Fig. 3 ETV5 controls glucosestimulated insulin secretion and exocytosis in INS-1 cells. (a) Total insulin content in INS-1 cells. (b) Glucose-stimulated insulin secretion following ETV5 knockdown. (c) The mitochondrial membrane potential response to $15 \mathrm{mmol} / \mathrm{l}$ glucose, as a percentage change in TMRE fluorescence. $(\mathbf{d}, \mathbf{e})$ Representative and averaged exocytotic responses at 2.5 or $15 \mathrm{mmol} / 1$ glucose, measured as cell capacitance increases during a train of ten membrane depolarisations. (f, $\mathbf{g})$ Representative voltage-activated $\mathrm{Ca}^{2+}$ currents and averaged $\mathrm{Ca}^{2+}$ charge entry in response to a single $500 \mathrm{~ms}$ membrane depolarisation. (h-j) Exocytotic response in human beta cells with 2.5 or $15 \mathrm{mmol} / 1$ glucose. (i)

Donor 1. (j) Donor 2. Black symbols and columns, NS siRNA control; white symbols and columns, ETV5 siRNA (siETV5); grey shading and outlines, stimulation at low glucose ( $2.5 \mathrm{mmol} / \mathrm{l})$ concentration; black shading and outlines, high glucose concentration $(15 \mathrm{mmol} / \mathrm{l})$. ${ }^{*} p<0.05$; ** $p<0.01$; $* * * p<0.001$
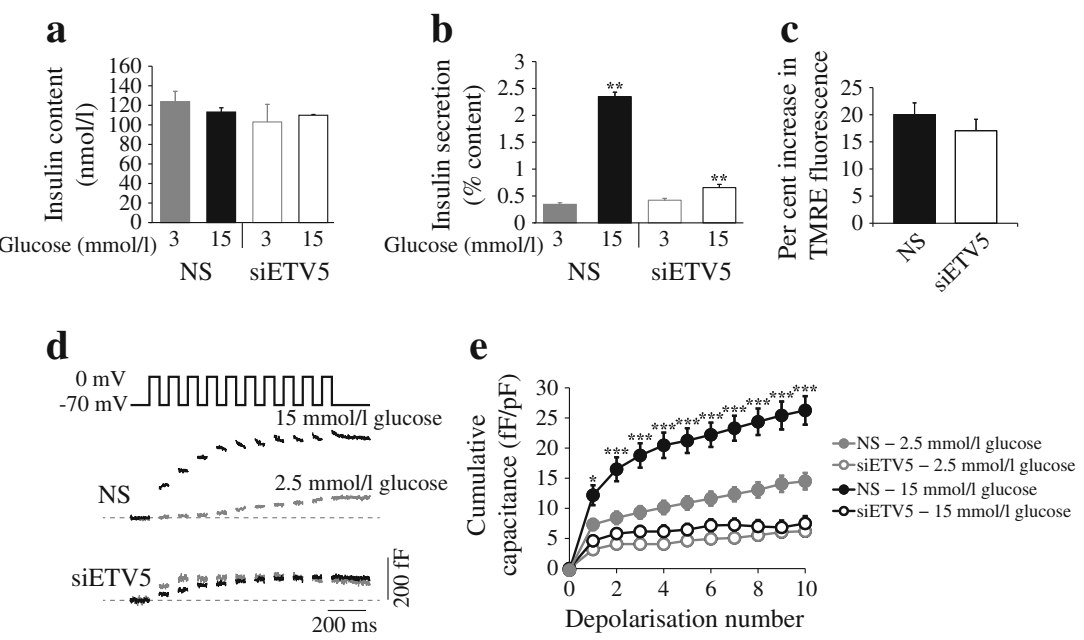

f
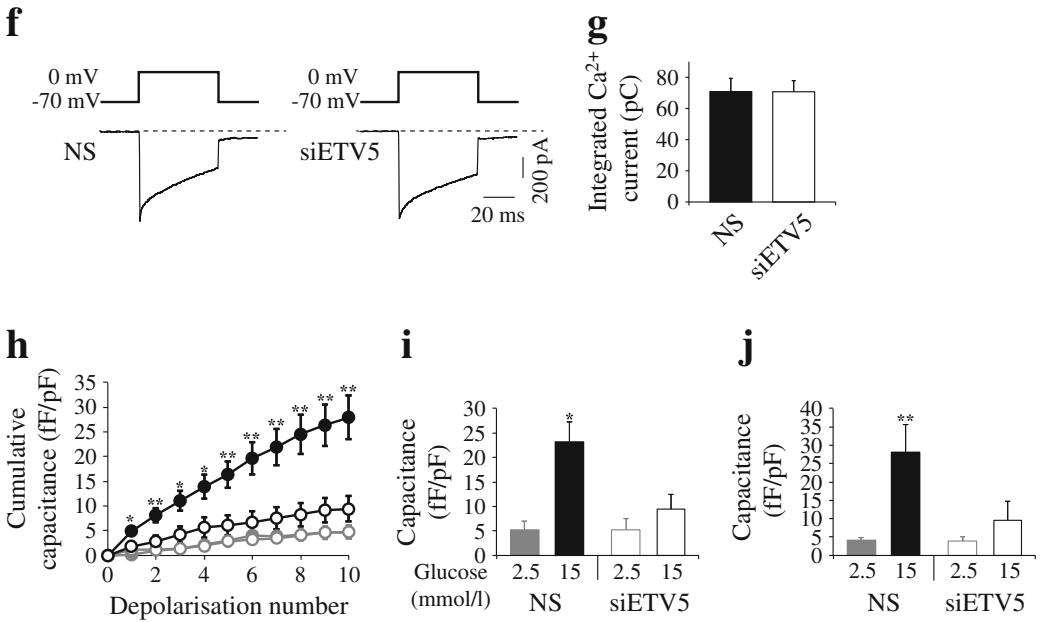

In a manner remarkably similar to what we observed in the INS-1 cells, the lack of ETV5 inhibited the exocytotic response of the human beta cells (Fig. 3h-j). These results are consistent with the in vivo data and provide strong evidence that ETV5 plays an important cell-autonomous role in GSIS.

ETV5 regulates genes implicated in insulin exocytosis To address how ETV5 impairs insulin exocytosis, we examined the expression of genes implicated in the exocytotic machinery in INS-1 cells. After $72 \mathrm{~h}$ of transfection with the siRNA (NS or Etv5), we performed a glucose-stimulated insulin secretion test and measured the transcripts. As expected, Etv5 expression was knocked down by $60 \%$ at $3 \mathrm{mmol} / 1$ glucose (data not shown) and $40 \%$ at $15 \mathrm{mmol} / \mathrm{l}$ (Fig. 4a). Figure $4 \mathrm{a}$ shows that the expression of Snap 25 (synaptosomal-associated protein 25), synaptotagmin VII and synaptotagmin IX were significantly downregulated in the absence of ETV5. However, the transcripts for syntaxin 1A, Munc-18 (mammalian uncoordinated-18) and Vamp2 (vesicleassociated membrane protein 2) were not modified by Etv5
siRNA. Moreover, protein levels of ETV5, synaptosomalassociated protein 25 (SNAP25), synaptotagmin VII and synaptotagmin IX were analysed by western blotting (Fig. 4b). The quantification of these blots showed a trend towards a reduction in ETV5 and SNAP25 levels, and a significant reduction for synaptotagmin IX in the presence of Etv5 siRNA (Fig. 4c). However, for synaptotagmin VII, it was impossible to quantify the protein level (data not shown).

\section{Discussion}

Recent GWAS studies have suggested ETV5 polymorphisms as genetic correlates of human obesity [9, 10]. Until now, precious little has been known about the role of ETV5 in metabolism. The present experiments were designed to elucidate the role of ETV5 in glucose regulation and BW.

The inactivation of ETV 5 resulted in reduced linear growth that was maintained through to 16 weeks of age. In addition to being shorter, the Etv5 $\mathrm{KO}$ mice weighed less, and this is 


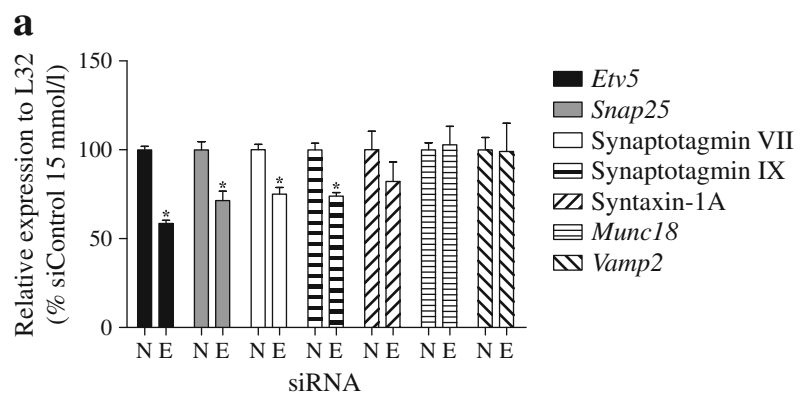

b

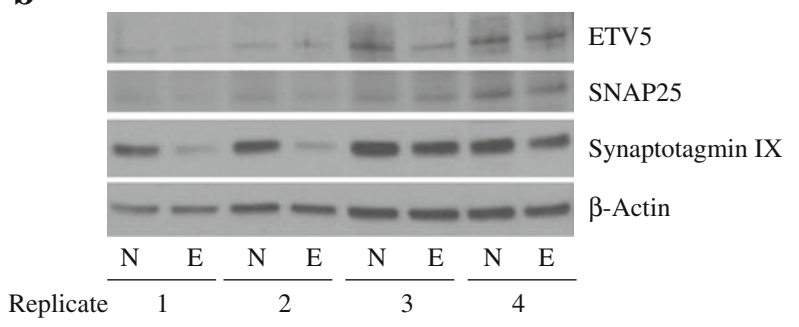

c

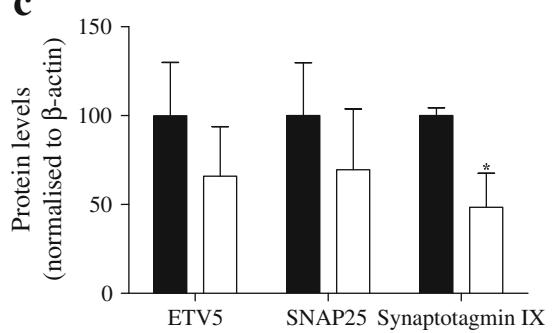

Fig. 4 Genes involved in the exocytotic mechanism. (a) Quantitative PCR analysis of RNA, (b) protein levels and (c) protein level quantification from INS-1 cells transfected with control $(\mathrm{N}=\mathrm{NS})$ or Etv5 $(\mathrm{E}=$ siEtv5 $)$ siRNA for $72 \mathrm{~h}$ and stimulated with glucose $(15 \mathrm{mmol} / \mathrm{l})$ for $1 \mathrm{~h}$. Black columns indicate NS siRNA control; white columns, Etv 5 siRNA. All values are given as means \pm SEM from $t$ test analysis. ${ }^{*} p<0.05$

partially the result of a reduced lean tissue mass $[15,16]$. However, KO mice also have reduced fat mass (Fig. 1a, b), and this is exacerbated when these mice are placed on an HFD (ESM Fig. 1c-e). The lean phenotype of Etv $5 \mathrm{KO}$ mice is a consequence of reduced food intake and not of increased $\mathrm{EE}$ (ESM Fig. 1f-i). Consistent with their reduced weight gain, KO mice had fewer and smaller adipocytes (ESM Fig. 1g) and, as expected, had reduced leptin levels (data not shown). After a prolonged fast, BG and insulin levels did not differ from levels in WT controls. However, after the animals had been re-fed, the BG levels remained similar whereas the insulin levels were reduced in the Etv5 KO mice, suggesting a reduced ability to secrete insulin (Fig. 1c, d). Unexpectedly, when challenged with glucose, the lean Etv5 $\mathrm{KO}$ mice were found to be severely glucose intolerant (Fig. 1e).

When we performed the ITTs, insulin sensitivity was actually improved in the Etv5 KO mice (Fig. 1f), ruling out the hypothesis that the impaired glucose regulation was secondary to insulin resistance as a result of an inability to make adequate adipose tissue. A hyperinsulinaemiceuglycaemic clamp would be needed to assess the specific role of the liver, adipose and skeletal muscle in the improved insulin sensitivity observed in the absence of ETV5.

Interestingly, fasting insulin levels were significantly lower in the Etv $5 \mathrm{KO}$ mice even though their BG levels were similar to those of control mice. However, the glucose levels of $\mathrm{KO}$ mice remained elevated for longer than those of their control littermates, consistent with an inability to mount a sufficient insulin response during the glucose challenge (Fig. 1e, g). Insulin clearance, however, appeared normal, and both genotypes had similar ratios of insulin to C-peptide (Fig. 1h). We first determined that the pancreatic weight was appropriate for the reduced BW in the KO mice. Furthermore, when the total insulin content was analysed, no differences were observed between genotypes, suggesting that the production of insulin was similar in WT and Etv $5 \mathrm{KO}$ mice (Fig. 2j).

Different growth factors, such as FGFs, have been implicated in the regulation of ETV5 levels in different organs such as the lung [17], kidney [18], musculoskeletal system [19] and spermatogonial stem cells [20]. FGF10 and epidermal growth factor have been shown to play a role in pancreatic ontogeny and regeneration via the stimulation of mitogen-activated protein kinase (MAPK) and Akt phosphorylation [21]. On the other hand, ETV5 is a target of MAPK [22], suggesting that ETV5 might be downstream of FGF10 signalling via MAPK activity. Moreover, it has been shown that ETV5 is present in the pancreas during all developmental stages [6], and elevated levels of its transcript (in the presence of FGF10 overexpression) could provoke progenitor arrest, organ hyperplasia and an inhibition of terminal differentiation $[7,8]$.

We therefore analysed the pancreatic morphology of Etv 5 $\mathrm{KO}$ mice and their controls. Etv $5 \mathrm{KO}$ mice had smaller islets and smaller beta cells (Fig. 2d-h), and it is known that a diminution in beta cell size can provoke a reduction in insulin production [23]. Therefore, Etv5 $\mathrm{KO}$ mice have a reduced beta cell size and a defect in glucose-stimulated insulin secretion, which together result in glucose intolerance. However, the defect in insulin secretion does not lead to frank diabetes, because it is accompanied by increased insulin sensitivity.

To avoid the confounding effect that ETV5 could have on beta cell development, and to assess whether this role of ETV5 in insulin secretion was a cell-autonomous effect in the beta cell, we analysed both a fully differentiated cell line and adult human islets in which we knocked down the expression of ETV5. We found that the lack of ETV5 in the beta cell lineage was associated with the same insulin levels, but that insulin secretion was impaired (Fig. 3a, b). However, both mitochondrial and $\mathrm{Ca}^{2+}$ channel activity remained intact (Fig. 3c, f, g). Nevertheless, reduced ETV5 specifically disrupts insulin 
granule exocytosis in INS-1 cells (Fig. 3d, e), as well as in human beta cells (Fig. $3 \mathrm{~h}-\mathrm{j}$ ). Unfortunately, it was not possible to analyse glucose-stimulated insulin secretion in the islets of WT and Etv 5 KO mice because of the difficulty of obtaining sufficient islets from the 129/Sv strain of mouse, either by perfusion or by collagenase inflation of the pancreas. Taken together, these data suggest that ETV5 plays a key role in insulin exocytosis in a cell-autonomous manner, and hence has a profound impact on insulin secretion, as a consequence causing severe glucose intolerance due to hypoinsulinaemia in the Etv $5 \mathrm{KO}$ mice.

Once we had identified the specific role that ETV5 plays in insulin exocytosis, we sought to identify genes from the exocytotic machinery regulated by ETV5. The exocytosis of insulin granules involves the formation of soluble $\mathrm{N}$-ethylmaleimide-sensitive factor attachment protein receptor (SNARE) complex, formed by VAMP2, syntaxin 1A and SNAP25 $[24,25]$. SNARE complex promotes fusion between the vesicle membrane and the plasma membrane upon $\mathrm{Ca}^{2+}$ influx [26]. Synaptotagmin VII and IX have been proposed to act as $\mathrm{Ca}^{2+}$ sensors in regulated exocytosis [27]. MUNC-18 is a protein that regulates the SNARE-assembly reaction by interacting with syntaxin $1 \mathrm{~A}$ and other SNARE proteins [25].

We observed that Snap25 and synaptotagmin VII and IX transcripts were downregulated (Fig. 4a) and only synaptotagmin IX protein levels (Fig. 4b, c) were significantly reduced in the absence of ETV5. Perinatal death occurs in Snap25 KO homozygous mice [28]. However, a missense mutant mouse showed impaired insulin granule priming, exocytosis and recycling in pancreatic beta cells [29]. In addition, synaptotagmin VII and synaptotagmin IX interact directly or indirectly with SNAP25 in pancreatic beta cells [30]. Taken together, these data suggest that, in the absence of ETV5, the genes coding SNAP25, synaptotagmin VII and synaptotagmin IX are downregulated, leading to a defect in fusion of the insulin vesicles to the plasma membrane, which provokes an impairment in insulin exocytosis.

It is important to generate $E t v 5$ beta cell-specific $\mathrm{KO}$ mice to analyse the direct impact of ETV5 in glucose homeostasis. The current work demonstrates for the first time that a total loss of function of ETV5 in mice results in reduced DIO and severe glucose intolerance; there is, however, no human association between ETV5 and glucose levels [31]. In conclusion, we demonstrate for the first time a clear role for ETV5 in metabolism. Despite the fact that Etv $5 \mathrm{KO}$ mice are lean, they are glucose intolerant due to hypoinsulinaemia, and have reduced beta cell size and impaired insulin exocytosis that occurs in a cell-autonomous fashion. Identifying ETV5 as a transcription factor that regulates genes implicated in insulin exocytosis is an unexpected outcome based on the GWAS studies. These data provide a new target for understanding the mechanisms of impaired insulin secretion that are critical in the development of type 2 diabetes.
Acknowledgements We thank K. M. Murphy, Washington University and Howard Hughes Medical Institute, for giving us the Etv $5 \mathrm{KO}$ mice, as well as the Assay Core of the Metabolic Diseases Institute of the University of Cincinnati for measurements of insulin and C-peptide. We also thank B. Grayson and A. Lewis, University of Cincinnati and M. Ferdoussi, University of Alberta, for discussions about this paper.

Funding This work was supported by the National Institutes of Health (grants DK54080, DK056863, DK093848 to RJS and DK017844 to $\mathrm{SCW}$ ). RJS is the corresponding author and guarantor. Work on exocytosis was supported by a grant to PEM from the Canadian Institutes of Health Research (MOP244739). MC holds a fellowship from Alberta Innovates - Health Solutions (AI-HS). PEM is an AI-HS Scholar and holds the Canada Research Chair in Islet Biology.

Duality of interest The authors declare that there is no duality of interest associated with this manuscript.

Contribution statement RGA contributed to the conception and design of the study and the acquisition, analysis and interpretation of the data, drafted/revised the article, wrote the manuscript, researched data and led the project. DHK, MC, X-QD, PTP, JP, AH, ED and JP contributed to the acquisition of the data and drafted/revised the article. DD'A, SCW, PEM and RJS contributed to the conception and design of the study and the analysis and interpretation of the data, and drafted/revised the article. All authors gave final approval of the manuscript to be published.

\section{References}

1. Hollenhorst PC, McIntosh LP, Graves BJ (2011) Genomic and biochemical insights into the specificity of ETS transcription factors. Annu Rev Biochem 80:437-471

2. Galang CK, Muller WJ, Foos G, Oshima RG, Hauser CA (2004) Changes in the expression of many Ets family transcription factors and of potential target genes in normal mammary tissue and tumors. J Biol Chem 279:11281-11292

3. Sementchenko VI, Watson DK (2000) Ets target genes: past, present and future. Oncogene 19:6533-6548

4. Sharrocks AD (2001) The ETS-domain transcription factor family. Nat Rev Mol Cell Biol 2:827-837

5. Gutierrez-Hartmann A, Duval DL, Bradford AP (2007) ETS transcription factors in endocrine systems. Trends Endocrinol Metab 18:150-158

6. Kobberup S, Nyeng P, Juhl K, Hutton J, Jensen J (2007) ETS-family genes in pancreatic development. Dev Dyn 236:3100-3110

7. Kobberup S, Schmerr M, Dang ML et al (2010) Conditional control of the differentiation competence of pancreatic endocrine and ductal cells by Fgf10. Mech Dev 127:220-234

8. Norgaard GA, Jensen JN, Jensen J (2003) FGF10 signaling maintains the pancreatic progenitor cell state revealing a novel role of Notch in organ development. Dev Biol 264:323-338

9. Thorleifsson G, Walters GB, Gudbjartsson DF et al (2009) Genomewide association yields new sequence variants at seven loci that associate with measures of obesity. Nat Genet 41:18-24

10. Willer CJ, Speliotes EK, Loos RJ et al (2009) Six new loci associated with body mass index highlight a neuronal influence on body weight regulation. Nat Genet 41:25-34

11. Chen C, Ouyang W, Grigura V et al (2005) ERM is required for transcriptional control of the spermatogonial stem cell niche. Nature 436:1030-1034

12. Hohmeier HE, Mulder H, Chen G, Henkel-Rieger R, Prentki M, Newgard CB (2000) Isolation of INS-1-derived cell lines with robust ATP-sensitive $\mathrm{K}+$ channel-dependent and -independent glucosestimulated insulin secretion. Diabetes 49:424-430 
13. Dai XQ, Plummer G, Casimir M et al (2011) SUMOylation regulates insulin exocytosis downstream of secretory granule docking in rodents and humans. Diabetes 60:838-847

14. Pfluger PT, Herranz D, Velasco-Miguel S, Serrano M, Tschop MH (2008) Sirt1 protects against high-fat diet-induced metabolic damage. Proc Natl Acad Sci U S A 105:9793-9798

15. Hippenmeyer S, Huber RM, Ladle DR, Murphy K, Arber S (2007) ETS transcription factor Erm controls subsynaptic gene expression in skeletal muscles. Neuron 55:726-740

16. Schlesser HN, Simon L, Hofmann MC et al (2008) Effects of ETV5 (ets variant gene 5) on testis and body growth, time course of spermatogonial stem cell loss, and fertility in mice. Biol Reprod 78: 483-489

17. Liu Y, Jiang H, Crawford HC, Hogan BL (2003) Role for ETS domain transcription factors Pea3/Erm in mouse lung development. Dev Biol 261:10-24

18. Lu BC, Cebrian C, Chi X et al (2009) Etv4 and Etv5 are required downstream of GDNF and Ret for kidney branching morphogenesis. Nat Genet 41:1295-1302

19. Brent AE, Tabin CJ (2004) FGF acts directly on the somitic tendon progenitors through the Ets transcription factors Pea3 and Erm to regulate scleraxis expression. Development 131: 3885-3896

20. Ishii K, Kanatsu-Shinohara M, Toyokuni S, Shinohara T (2012) FGF2 mediates mouse spermatogonial stem cell self-renewal via upregulation of Etv5 and Bcl6b through MAP2K1 activation. Development 139:1734-1743

21. Kayali AG, Stotland A, Gunst KV et al (2005) Growth factor-induced signaling of the pancreatic epithelium. J Endocrinol 185:45-56
22. Janknecht R, Monte D, Baert JL, de Launoit Y (1996) The ETSrelated transcription factor ERM is a nuclear target of signaling cascades involving MAPK and PKA. Oncogene 13:1745-1754

23. Giordano E, Cirulli V, Bosco D, Rouiller D, Halban P, Meda P (1993) $B$ cell size influences glucose-stimulated insulin secretion. Am J Physiol 265:C358-C364

24. Eliasson L, Abdulkader F, Braun M, Galvanovskis J, Hoppa MB, Rorsman P (2008) Novel aspects of the molecular mechanisms controlling insulin secretion. J Physiol 586:3313-3324

25. Lang T, Jahn R (2008) Core proteins of the secretory machinery. Handb Exp Pharmacol 184:107-127

26. Gerber SH, Sudhof TC (2002) Molecular determinants of regulated exocytosis. Diabetes 51(Suppl 1):S3-S11

27. Gauthier BR, Wollheim CB (2008) Synaptotagmins bind calcium to release insulin. Am J Physiol Endocrinol Metab 295:E1279-E1286

28. Washbourne P, Thompson PM, Carta M et al (2002) Genetic ablation of the t-SNARE SNAP-25 distinguishes mechanisms of neuroexocytosis. Nat Neurosci 5:19-26

29. Jeans AF, Oliver PL, Johnson R et al (2007) A dominant mutation in Snap25 causes impaired vesicle trafficking, sensorimotor gating, and ataxia in the blind-drunk mouse. Proc Natl Acad Sci U S A 104: 2431-2436

30. Boal F, Laguerre M, Milochau A, Lang J, Scotti PA (2011) A charged prominence in the linker domain of the cysteine-string protein Cspalpha mediates its regulated interaction with the calcium sensor synaptotagmin 9 during exocytosis. FASEB J 25:132-143

31. Speliotes EK, Willer CJ, Berndt SI et al (2010) Association analyses of 249,796 individuals reveal 18 new loci associated with body mass index. Nat Genet 42:937-948 